\title{
Miscellaneous
}

\author{
Jan-Felix Müller*
}

\section{Compatibilist Libertarianism: Advantages and Challenges (Conference Report)}

\author{
https://doi.org/10.1515/krt-2021-0035
}

Published online December 23, 2021

The traditional way to classify philosophical positions in the free will debate is to ask whether or not they regard free will as compatible with determinism. Compatibilists state such a compatibility, whereas incompatibilists deny it. Libertarians are those incompatibilists who postulate the actual existence of free will (and are thus committed to deny the truth of determinism). According to this classical picture, compatibilism and libertarianism are mutually exclusive positions because their definitions involve contrary answers to the question whether free will is compatible with determinism. Still, in recent years a mediating position called compatibilist libertarianism has been proposed. Its main idea is to differentiate between different kinds of determinism, or between determinism at different ontological levels. Free will is claimed to be compatible with one kind, but incompatible with another kind of determinism. If, against this background, free will is claimed to actually exist, then one gets a libertarian position with respect to the freedom-incompatible kind of determinism, but a compatibilist position with respect to the other kind of determinism.

On October 29, 2021, an online workshop called "Compatibilist Libertarianism: Advantages and Challenges”, organized by Alexander Gebharter (LMU Munich) and Maria Sekatskaya (HHU Düsseldorf), served the detailed discussion of this approach. It focused on the specific account of Christian List (2014, 2019b), who developed and prominently defended compatibilist libertarianism. List also introduced the conference with a presentation of his account, before seven further talks were concerned with its critical discussion. In what follows, I will paraphrase some main ideas of the conference talks, and end with highlighting a philosophical dissent that seemed to pervade several discussions. The structure of my report does not mirror the temporal order of the presentations at the conference.

\footnotetext{
*Corresponding author: Jan-Felix Müller, Institut für Philosophie, Leibniz Universität Hannover, Im Moore 21 (Hinterhaus), 30167 Hannover, Germany, E-mail: jan-felix.mueller@philos.unihannover.de
} 
In his introductory presentation, Christian List (LMU Munich) lays down his motivation for developing compatibilist libertarianism as follows: He wants to account for the existence of free will in a way that is compatible with physical determinism, but he still does not want to deny (as many compatibilists do) that the ability to act otherwise is necessary for free will. The problem, however, is that such an ability to act otherwise appears absent if one examines a deterministic physical structure of the world. But, List argues, just because things are undetectable at the physical level, this does not mean that they aren't real. After all, political institutions are not detectable at the physical level as well, but we might still have very good reason to regard them as real phenomena on a higher ontological level than the physical. Similarly, we should regard agents' alternative possibilities as real on an agential level. This is, List argues, because they are indispensable for many social explanations, and we are usually justified to believe in the existence of what is explanatorily indispensable. This leads to the following picture: There are alternative possibilities at the agential level, although there need be no alternative possibilities at the physical level.

How is that possible? List's explanation refers to a unidirectional supervenience relation between the agential and the physical level. Agential states supervene on physical states, but not vice versa: qualitatively different physical states could be realizers of one and the same agential state. If physical determinism holds, each possible physical realizer-state determines its physical consequences. But as long as the different possible physical realizer-states of one agential state determine different consequences, there need be no determinism at the agential level. For a given agential state could be realized by several physical states, and if these different physical states determine grossly different consequences, the agential state itself does not determine its consequences-simply because very different physical histories are compatible with the respective agential state. This way, there can be determinism at the physical level and still indeterminism at the agential level, which allows for alternative agential possibilities. Therefore, even if one regards alternative possibilities as necessary for free will, one can still regard free will as compatible with determinism at the physical level. For all that would undermine the agent's ability to act otherwise is determinism at the agential level, and such agential determinism need not hold merely because there is physical determinism. So, we have exactly what is characteristic for compatibilist libertarianism: Free will is supposed to exist and to be compatible with one kind of determinism, namely physical determinism, but incompatible with another kind of determinism, namely agential determinism (cf. List 2014, 2019b).

Leonhard Menges (University of Salzburg) criticizes List's argument for the reality of free will. As sketched above, List argues that alternative agential possibilities are explanatorily indispensable for free will, and that we are therefore 
justified to believe in their existence. Slightly more specific, the alternative agential possibilities are argued to be explanatorily indispensable because in order to make sense of someone's actions (in the social sciences), we usually need to interpret the respective person as having faced a choice between the finally chosen action and alternative available options. Since we are justified to believe in the reality of these explanatorily indispensable alternative possibilities of the agent, List argues, there is no problem with requiring alternative agential possibilities for free will.

But as Menges points out, there are different senses in which an "ability to act otherwise" can be ascribed to an agent. The ability to act otherwise that List wants to be established is the ability to act otherwise that is necessary for free will. Since free will is deeply connected to moral responsibility, we can also understand this ability as the ability to act otherwise which is necessary for moral praise- and blameworthiness. It is not clear, however, whether this freedom-necessary ability to act otherwise is identical to the explanatorily necessary ability to act otherwise for which List argues that we are justified to believe in its existence.

List's argument relies on the assumption that both abilities to act otherwise are identical. But this implicit premise, Menges claims, is not plausible. In order to argue for this claim, he construes a scenario in which an elective decision is psychologically examined. In order to make sense of the psychological investigation, we should assume that the voters have an ability to act otherwise in the sense that is explanatorily required. But Menges claims that the elective decisions might still be unfree and lack the ability to act otherwise that is necessary for praise- and blameworthiness, for example if the voters had been systematically manipulated. Summing Menges's position up, List's argument requires the identification of two different kinds of ability to act otherwise (namely the freedom-necessary and the explanatorily necessary one), but this identification is not adequately justified. If so, List's argument relies on an implicit premise with lacking or dubious justification.

The presentation of Alfred R. Mele (Florida State University) raises a luck problem for List's compatibilist libertarianism. Usually, luck problems arise for libertarian accounts which require indeterminism in order to account for an agent's free control over her actions. Such luck problems for libertarianism question whether indeterminism really allows for more agential control than determinism (as the libertarians claim), or whether indeterminacies rather make the respectively undetermined outcomes a matter of mere luck. For one might worry that if an action is undetermined, whether it occurs is unsettled and thus less (rather than more) under the agent's control than a determined action. Mele emphasizes that he does not intend to present an argument to the conclusion that undetermined actions are in fact merely a matter of luck. Rather, he merely wants to raise a question to which libertarians are challenged to find an answer: How is it supposed to be possible that freedom-establishing undetermined actions are not merely a matter of luck? 
How does this luck problem for libertarianism connect to compatibilist libertarianism? List's compatibilist libertarian account differs from traditional libertarian accounts in that it does not require the absence of physical determination for any events, so there need be nothing unsettled at the physical level. Still, List requires the absence of determinism at the agential level, so some of an agent's actions must not be determined by her earlier agential states. Admittedly, there can be determinants of the action's physical realizers, but it's an essential part of List's theory to not regard determination at the physical level as relevant to the agent's freedom. Therefore, List's theory appears to face the same luck problem at the agential level that traditional libertarians face at the physical level.

List's response to the luck problem rests on a differentiation between possible and rational outcomes. He argues that just because several outcomes are agentially possible, not more than one of them needs to be rational. If such a uniquely rational option is chosen, this decision is not merely lucky or arbitrary because it is singled out by rationality considerations. And this response to the luck problem is still compatible with requiring the ability to act otherwise, because what counts for this ability are not only rational possibilities but all agential possibilities. The resulting picture is that although many options are agentially available, only one of them needs to be singled out as rational, and in this case the choice of the rational option is not arbitrary (cf. List 2014: 172-173, 2019b: 108-111).

This response was critically discussed. A problem it faces is that although it singles out certain options as rational and thus as non-arbitrary, one might question whether it also accounts for the agent's control in choosing this option. For one might criticize that whether the agent indeed chooses the rational option is left open on the agential level, such that it is unclear how the agent could be able to control her final decision for the rational option (cf. Mele 2020).

Nadine Elzein (University of Warwick) defends List's conception against this luck objection. In order to do so, she criticizes the luck objection's underlying demand for contrastive explanations: The above-mentioned worry seems to be that if it is left open on the agential level which option the agent finally chooses, there is no agent-level explanation for why the agent chooses the way she actually does rather than in another way that is also compatible with her agential history. Elzein argues that the availability of such contrastive explanations is not necessary for free decisions, and so the luck problem need not threaten (compatibilist) libertarianism.

First, we should note an important respect in which Elzein departs from List's conception. What List regards as necessary for free will are alternative agential possibilities of any kind. Elzein restricts her focus on rational possibilities. She argues for this restriction by pointing to the irrelevance of irrational alternatives for moral blame: you can only be blamed if you fail to decide for a rational alternative. 
From this perspective, even the demand for alternative possibilities might be understood as a demand for alternative rational possibilities.

Now imagine a situation in which this requirement is satisfied: someone is faced with a decision between two different rational options. In order for both options to be genuinely rational, they should be rationally on a par. But in this case, there is no rational (pro toto) reason to prefer one option over the other. So, if there is a contrastive explanation available for the agent's decision between the two options, then this contrastive explanation cannot refer to an overall consideration of rationality. Rather, the contrastive explanation has to refer to non-rational elements. But why should one demand contrastive explanations of such a non-rational kind for moral responsibility or free will? While a purely rational decision might be burdened with some degree of arbitrariness if the best available options are rationally on a pair, this arbitrariness only seems to increase (rather than decrease) if the final decision is settled by non-rational elements. To use Elzein's example, if one has to make a hiring decision between two equally qualified applicants and no hiring option is rationally superior, making the final decision between both options on the basis of a non-rational element like a racist bias is something that undermines one's rationality. And something that undermines one's rationality should not be regarded as a necessary condition for exercising free will.

On the basis of these considerations, Elzein rejects the demand for contrastive explanations. If one follows her reasoning, the lack of contrastive agential explanations in the conception of compatibilist libertarianism need not be regarded as a problem. What is more, even traditional libertarian conceptions would not be threatened by the lack of contrastive explanations anymore, simply because the availability of such contrastive explanations need not be regarded as necessary for free will.

Several talks of the conference are concerned with how exactly to understand the relation between agential and physical alternative possibilities in List's theory. As mentioned above, List argues that there can be alternative possibilities at the agential level without this requiring alternative possibilities at the physical level. This conception is criticized by Tuomas K. Pernu (University of Eastern Finland), whose presentation involves a comparison between List's compatibilist libertarianism and Jerry Fodor's multiple-realization argument against (bridgelaw) reduction of psychology. Fodor (1974) argues that psychological regularities need not have structurally similar counterparts at the physical level because psychologically identical phenomena can be realized by multiple, physically heterogenous phenomena. If this is the case, (informally speaking) relevant psychological patterns seem to disappear if one switches perspective from the psychological to the physical level. 
This picture might appear similar to List's view, which also crucially relies on the assumption that identical agential states can be realized by multiple and heterogeneous physical states. However, Pernu points out a crucial difference between Fodor's view on the one hand and List's compatibilist libertarianism on the other hand. On Fodor's view, a deterministic relation at the psychological level could be realized by several heterogeneous deterministic relations at the physical level. On List's view, there are also several possibilities for deterministic relations at the physical level, but their heterogeneity is supposed to account for the lack of deterministic structure at the agential level.

Pernu presents a problem for this view. For illustration, imagine a particular case in which an agential state $A_{1}$ is supposed to leave it undetermined whether the agential state $A_{2}$ or the incompatible agential state $A_{3}$ occurs at a later time $t$. Now consider the following: $A_{1}$ has to be realized by some physical state $P_{1}$. (There might be several possible physical realizers, but only one possible realizer, namely $P_{1}$, is in fact the actual realizer.) If there is determinism at the physical level, $P_{1}$ determines some other physical state for time $t$. This state $P_{t}$ (vertically) determines the agential state that it realizes, which is either $A_{2}$ or $A_{3}$. Let's say $P_{t}$ determines $A_{3}$. Since $P_{1}$ determines $P_{t}$, and $P_{t}$ determines $A_{3}, P_{1}$ also determines $A_{3}$. But this appears to conflict with the claim that $A_{1}$ does not determine $A_{3}$. After all, $A_{1}$ is physically realized (and vertically determined) by $P_{1}$, and $P_{1}$ does determine $A_{3}$. Against the background of these considerations, the "indeterminacies" at the agential level appear illusory. For if an earlier agential state is claimed to leave it undetermined which later agential state occurs, the impression of indeterminacy disappears as soon as one takes the physical realizer (of the earlier agential state) into account.

Pernu also sketches a possible escape from this problem. The above considerations assume that identical physical states (vertically) determine identical agential states. (This assumption is in fact implied by the claim that agential states supervene on physical states.) But now imagine instead a "reversed Fodor structure", according to which identical states at the lower physical level can be multiply "realized" (metaphorically speaking) by heterogenous states at the higher agential level. In this case, determinism at the lower physical level need be no threat to free will because determined physical effects are still compatible with different agential states. In other words, there can be alternative possibilities about which agential state "realizes" a given physical state even if this physical state itself is determined. Giving up the vertical determination of agential states by physical states leads therefore to a conception in which alternative agential possibilities are indeed compatible with determinism at the physical level. Note, however, that this sketched conception differs from List's view in that it rejects the supervenience of the agential level on the physical level, whereas List explicitly demands this supervenience. 
List's claim that agential alternatives are compatible with physical determinism is also criticized by Alexander Gebharter (LMU Munich), who presents his so-called "Collapse Argument" against compatibilist libertarianism. The main idea is that, under physical determinism, the appearance of alternative agential possibilities collapses as soon as one considers that a given agential state is always realized by a certain physical state. For if this physical state determines its physical consequences, then also the later agential states (which supervene on these physical consequences) are eventually fixed by the original physical state in which the agent is located. One might object that what fixes the future agential states are not the agential properties of the original agential state, but rather the physical properties of the underlying physical state. But since these physical properties are still fixed in the original situation of the agent, this doesn't change anything about the agent's lack of genuine alternative possibilities (cf. Gebharter 2020).

Until here, the objections of Gebharter and Pernu appear closely similar. What is more, both philosophers suggest similar remedies to the problem(s) that they raise for List's conception: both suggest to weaken the demand for supervenience (of the agential on the physical level). But whereas Pernu suggests to completely abandon the supervenience demand, Gebharter suggests a modification towards a probabilistic version of supervenience. Traditional supervenience requires that if two systems differ in their supervening (here: agential) properties, then they always differ in their subvening (here: physical) properties as well. In contrast, Gebharter's weakened probabilistic version of supervenience only demands that if two systems differ in their supervening properties, then they must have different probabilities of having each subvening property. (This probabilistic weakening is claimed to be comparable to the way in which many current theories of causation do not require exceptionless regularities anymore, but are content with relying on probabilistic correlations.)

This proposal has the advantage that it does not completely abandon the requirement that agential states depend on physical states. Rather, it merely weakens this dependence requirement, such that there can be qualitatively different agential states with qualitatively identical physical realizers-but only if the relevant probabilistic indeterminacies are settled the right way. Still, this conception allows for the compatibility of alternative agential possibilities with physical determinism, because even if a physical state is determined, there can still be alternative possibilities about which agential state this physical state realizes. This suggested response to the collapse problem might be regarded as libertarian in spirit, because it postulates that agential states are undetermined not only by earlier agential states (as on List's view), but also by their underlying physical states. 
Maria Sekatskaya (HHU Düsseldorf) also relies on Gebharter's Collapse Argument, but the remedy for compatibilist libertarianism that she proposes is compatibilist (rather than libertarian) in spirit. Her talk starts with a summary of the controversy between classical compatibilists and libertarians. Both usually require the ability to act otherwise for free will. But when examining whether an agent in fact has the required ability to act otherwise, they usually disagree about which possible scenarios count for this ability-or, in other words, which possible worlds are accessible to an agent. Libertarians usually postulate that a world is only accessible to an agent (at time $t$ ) if this world completely equals the agent's actual world in its laws and in its history (up to time $t$ ). This so-called actualist conception of accessibility leads to incompatibilism about free will and the ability to act otherwise. For if determinism holds, all worlds with the same laws and past as the actual world also have (by definition of "determinism") the same future, such that no worlds with a different future than the actual world are accessible.

Classical compatibilists avoid this conclusion by rejecting the actualist conception of accessibility. They regard some worlds as accessible although these worlds involve slight divergences from the actual world's laws and/or past. For example, if one analyses the ability to act otherwise in terms of what one would have done if one had wanted otherwise, then one claims that there is an accessible world which differs from the actual world's past in respect of what the agent wanted. Such accessible worlds with different pasts or laws can involve different futures than the actual world, and therefore classical compatibilists can account for the ability to act otherwise even if the actual world's future is determined. However, the burden of this strategy is that it appears intuitively implausible to regard worlds with different laws or pasts as accessible, simply because the laws and the past appear as fixed and not up to the agent (anymore).

How should one locate List's compatibilist libertarianism in this debate about accessibility? Prima facie, it seems that List's conception can reconcile an actualist understanding of accessibility with compatibilism about alternative possibilities and physical determinism. For the different scenarios which ground the agent's ability to act otherwise are required to be completely identical with respect to their (agential) past, and no scenario violates the natural laws. And still, the alternative agential possibilities are claimed to be compatible with determinism on the physical level.

Unfortunately, this compatibility claim is threatened by Gebharter's Collapse Argument, which shows that taking into account the agential state's physical realizer leads to a collapse of the alleged alternative agential possibilities. Sekatskaya suggests that this collapse problem can be avoided by weakening the actualist notion of accessibility. For consider why exactly the collapse problem occurs: if one regards as accessible only worlds with the same past and laws as the 
actual world, all accessible worlds have not only the same agential past but also the same physical realization of this past, such that physical determinism leads to the collapse of alternative possibilities. But if one regarded as accessible not only worlds with exactly this past, but also worlds with a slightly different past, then this problem can be circumvented. For if the accessible worlds can differ in their realizing physical properties, then they can also differ in their determined physical consequences. And if these differences regarding the physical consequences are large enough to make a difference at the agential level, then the alternative agential possibilities are accounted for.

One might object that this proposal involves rejecting the actualist notion of accessibility, and that it is therefore no more plausible than classical compatibilism. But Sekatskaya has a response to this objection. Remember that List's conception involves a unidirectional supervenience relation of the agential on the physical level, such that agential states are multiply realizable by physical states. This allows for the possibility that the different accessible worlds which feature in Sekatskaya's proposal can be identical in their agential histories although they differ in their physical histories.

Admittedly, Sekatskaya's proposal indeed conflicts with the libertarian's original actualist notion of accessibility. This is because it involves the accessibility of worlds which differ from the actual world in respect of their physical histories. But because of the multiple realizability of agential states, Sekatskaya's proposal is still compatible with the requirement that all accessible worlds are identical to the actual world in their agential histories. In this case, one can maintain an agentially actualist notion of accessibility, according to which only worlds with the same laws and the same agential history as the actual world are accessible. Sekatskaya argues that this way of only cautiously weakening the actualist notion of accessibility might be more plausible than completely rejecting it. (Completely rejecting the actualist notion of accessibility would even be compatible with allowing for the accessibility of worlds with different agential histories, or with different laws, and many traditional classical compatibilists do indeed allow for just this.)

Sekatskaya's approach aims to stick as closely as possible to libertarian actualist requirements. But since a completely actualist notion of accessibility doesn't allow for the compatibility of physical determinism and the agential ability to act otherwise (as Gebharter's Collapse Argument shows), an agentially actualist notion of accessibility appears to be the best that one can get (if one wants to account for the above-mentioned compatibility). This way, Sekatskaya's proposal shows how one might get from List's compatibilist libertarianism to an improved version of classical compatibilism.

As explained, Gebharter and Sekatskaya present two different approaches to remedy the problem that the Collapse Argument poses for List's compatibilist 
libertarianism. List, however, in his response to these two presentations (and also in response to the presentation of Tuomas K. Pernu, who raises a very similar problem), denies that there is a need for such a remedy. He admits that if one did decide to take the agential state's underlying physical realizers into consideration, then these physical realizers would indeed yield the agent's future actions. But List denies that it is reasonable to take the physical realizers into account in the first place. On his view, if one considers whether an agent is able to do otherwise, one should restrict one's considerations to the agential level. What justifies this restriction of view, List argues, is that the agential and the physical level are not merely different perspectives on one and the same thing. Rather, List ascribes an own ontological status to the agential level, and this ontological status is supposed to be irreducible to the ontological status of the physical level. What justifies him in assuming this irreducible status, as briefly indicated above, is the explanatory indispensability of the agential level, and the "naturalistic ontological attitude" which (under usual circumstances) certifies one's belief in the reality of what is explanatorily indispensable.

If one grants that the agential level has such an irreducible ontological status, then it's just this agential level which is relevant for the question whether agents have alternative possibilities, List seems to argue. Taking into account the ontologically different physical level would merely distract from what is really relevant for free-will considerations. Accordingly, there is no need to worry about the consequences of taking the agential level's physical realizers into account-one simply shouldn't take them into account in the first place. A very similar idea is also important for List's response to a challenge that Taylor W. Cyr raises.

Taylor W. Cyr (Samford University), in his presentation of joint work with Parker Gilley, examines how List's compatibilist libertarianism relates to Peter van Inwagen's (1983) third version of the “Consequence Argument”. This argument is supposed to formally show that (physical) determinism undermines any agent's ability to act otherwise. Very simplified, it does so by introducing a modal "no choice" operator, which applies to any true proposition about which no one ever has a choice. If determinism is true, this no-choice operator applies to a conditional which has the complete description of the natural laws and of a distant past state (before there were any agents) in its antecedent, and the description of some allegedly free action in its consequent. The no-choice operator seems to also apply to the description of the natural laws and of the distant past state. Now the argument relies on an inference rule called $\beta$, which says that if the no-choice operator applies to a conditional and to this conditional's antecedent, then it must also apply to this conditional's consequent. This yields the conclusion that the no-choice operator applies to the description of the allegedly free action. And since the argument is independent of which specific 
action one considers, the same reasoning can be used to show that if determinism is true, no one ever has a choice about any action.

If one denies this conclusion, one should be able to explain where exactly one departs from the Consequence Argument. Cyr argues that List's compatibilist libertarianism is incompatible with the soundness of the Consequence Argument, but that List doesn't explicitly argue against any premise of it. But if List's position is supposed to be convincing, Cyr argues, List should be able to do just this: he should be able to specify with which premise of the Consequence Argument he disagrees, and he should be able to argue against it.

In his response to Cyr's presentation, List doesn't explicitly point to an individual premise of the Consequence Argument that he rejects, but he rather raises a more general objection against van Inwagen's argument. Remember that List regards the agential and the physical level as ontologically distinct. Due to this distinctness, he argues, it is inappropriate to assign a no-choice operator to propositions about physical laws or about micro-physical states. For these propositions concern the physical level, whereas choice (with which the no-choice operator is concerned) is not a physical but an agential concept. Thus, List claims that van Inwagen's assignment of the no-choice operator to certain physical propositions constitutes a category mistake (cf. List 2019a).

This seems to reveal a broader philosophical dissent, which also shows up in List's above-mentioned response to Pernu's, Gebharter's and Sekatskaya's presentations. List seems to argue that if we are engaging in free-will considerations, the ontological separateness of the agential level allows us to disregard certain phenomena at the physical level. In contrast, other participants of the conference still insist on taking exactly these physical phenomena into account. It would be interesting to further investigate what exactly could justify List in separating the agential from the physical level in such a strong way that phenomena on the physical level are not only regarded as ontologically separate from agential phenomena, but moreover as not even relevant to certain free-will considerations.

Acknowledgments: Thanks to everyone who contributed to the success of the conference, especially to Alexander Gebharter and Maria Sekatskaya for organization, to Christian List for presenting and defending the discussed account of compatibilist libertarianism, to Taylor W. Cyr, Nadine Elzein, Alfred R. Mele, Leonhard Menges, and Tuomas K. Pernu for presenting and discussing their critical work on this account, and to all participants for their interest and their discussions. Besides these general acknowledgments, I would like to personally thank Alexander Gebharter for offering me the opportunity to write this conference report. I am also indebted to Maria Sekatskaya for her proofreading, to Leonhard Menges, Alexander Gebharter, and Maria Sekatskaya for their feedback, and to all 
presenters who provided me with their presentation slides, handouts, and/or background papers.

Conference webpage: A timetable of the conference and short abstracts of the talks can be found at https://www.mcmp.philosophie.uni-muenchen.de/events/ workshops/container/comp_lib_21/index.html.

\section{References}

Fodor, J. A. 1974. "Special Sciences (Or: The Disunity of Science as a Working Hypothesis)." Synthese 28 (2): 97-115.

Gebharter, A. 2020. “Free Will as a Higher-Level Phenomenon?” Thought-A Journal of Philosophy 9 (3): 177-87.

List, C. 2014. "Free Will, Determinism, and the Possibility of Doing Otherwise." Noûs 48 (1): 156-78.

List, C. 2019a. "What's Wrong with the Consequence Argument: A Compatibilist Libertarian Response." Proceedings of the Aristotelian Society 119 (3): 253-74.

List, C. 2019b. Why Free Will is Real. Cambridge: Harvard University Press.

Mele, A. R. 2020. “Free Will and Luck: Compatibilism versus Incompatibilism.” The Monist 103 (3): 262-77.

van Inwagen, P. 1983. An Essay on Free Will. Oxford: Oxford University Press. 